Regards sur l'économie allemande

Bulletin économique du CIRAC

116-117| 2015

Varia

\title{
Banque centrale européenne : d'une légitimité de crise à une crise de légitimité ?
}

\section{Alain Fabre}

\section{OpenEdition}

\section{Journals}

Édition électronique

URL : http://journals.openedition.org/rea/4816

DOI : $10.4000 /$ rea.4816

ISSN : 1965-0787

Éditeur

CIRAC

Édition imprimée

Date de publication : 1 juillet 2015

Pagination : 43-54

ISSN : 1156-8992

\section{Référence électronique}

Alain Fabre, «Banque centrale européenne : d'une légitimité de crise à une crise de légitimité ? »,

Regards sur l'économie allemande [En ligne], 116-117 | juillet 2015, mis en ligne le 01 juillet 2017, consulté le 03 mai 2019. URL : http://journals.openedition.org/rea/4816 ; DOI : 10.4000/rea.4816 


\section{Banque centrale européenne : d'une légitimité de crise à une crise de légitimité ?}

\author{
Alain Fabre
}

La Banque centrale européenne (BCE), telle qu'elle se présente après la crise des dettes souveraines, offre un visage bien différent de ce qu'il fut jusqu'en 2007. De la chute de Lehman Brothers en septembre 2008 au début de la crise grecque (2009-2010), la BCE s'est trouvée face à une crise d'une gravité sans précédent qui portait en elle, notamment en 2011, le risque d'une implosion de l'Euro. Face aux gouvernements et aux marchés, elle s'est imposée comme le rempart de la stabilité monétaire et financière.

La crise a mis au grand jour les illusions et les contradictions de la construction d'origine. La BCE a dû se réinventer à partir d'une gestion habile de ses statuts. Au terme de cette transformation, elle est devenue une banque centrale de plein exercice - ce qui était loin d'être le cas avant la crise -, au point de revendiquer désormais sans complexe sa fonction de prêteur en dernier ressort bancaire. Cette phase a consacré son autorité et sa crédibilité. Mais l'adoption, annoncée depuis de nombreux mois, explicite depuis janvier 2015, d'une politique de Quantitative Easing (QE), pointe extrême des techniques dites "non conventionnelles", pourrait bien créer de nouvelles équivoques lourdes de risques pour elle-même et pour l'avenir de l'Euro.

\section{Une monnaie unique implique union de transferts, union budgétaire et union bancaire}

La crise des dettes souveraines a mis à nu les contradictions initiales de la monnaie unique. La zone Euro a en effet été construite sur une triple contradiction : une union monétaire n'est viable ni sans transferts entre les Etats qui la composent, ni sans un contrôle des politiques de chacun d'entre eux par tous, ni non plus sans une union bancaire pour éviter les risques de sa fragmentation.

La première contradiction est qu'il ne peut y avoir d'union monétaire sans solidarité de tous au bénéfice de chacun. On a assez dit à juste titre que l'Union monétaire n'était pas une zone monétaire optimale : formulée par Robert Mundell (1960), celle-ci définit une zone monétaire dans laquelle les parties qui la composent ont avantage à adopter une monnaie unique, ce qui suppose une parfaite mobilité des facteurs de production (travail et capital). Mais les EtatsUnis ou l'Allemagne ne le sont pas plus si on entend par là une homogénéité des niveaux de vie et de productivité entre zones économiques qui adoptent une monnaie unique. En Allemagne, exemple majeur de zones uniques disparates liées par une monnaie unique, la réunification a permis de vérifier que monnaie unique et transferts entre zones constituaient deux faces de la même médaille.

La crise des dettes souveraines a montré que l'union de transferts s'imposait d'elle-même, en conférant aux parlementaires grecs, portugais, irlandais, le pouvoir de lever des impôts sur les contribuables de toute l'Union monétaire, lesquels ont été placés devant l'alternative brutale mais vraie de l'obligation de solidarité (même si elle est et doit être conditionnelle) ou de la dislocation de la monnaie unique. En réalité, cette solidarité est activée et curieusement amplifiée par l'absence d'échelon fédéral : la dette des uns est la dette de tous. Dans un système

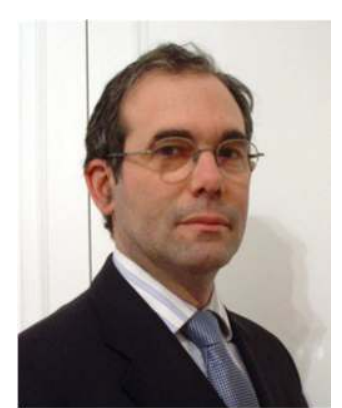

Alain Fabre, Conseil financier d'entreprises, Expert économique à la Fondation Robert Schuman et à l'institut de l'Entreprise

1. Union de transferts

Solidarité

et absence d'échelon fédéral 
2. Union budgétaire...

... mais concurrence fiscale et sociale

Lecture erronée des déséquilibres

Double impératif : intégration budgétaire et réformes structurelles

3. Union bancaire fédéral, seule la dette de l'instance fédérale bénéficie du statut d'actif sans risque. En zone Euro, tous les Etats 'fédérés' par la monnaie unique ont bénéficié de ce statut au moins avant que la crise vienne percuter cet édifice.

La deuxième contradiction de l'Euro en version d'avant crise est qu'il n'est pas possible d'instituer une monnaie unique et de maintenir une autonomie totale des politiques budgétaires. Une décision unilatérale de politique budgétaire de l'un des Etats membres exerce des effets - expansifs ou restrictifs - sur ses partenaires, alors même que l'ajustement des déficits d'un côté et des excédents de l'autre ne peut pas être opéré par la modification des parités de change. Cette autonomie s'entend d'ailleurs au-delà de la seule politique budgétaire : droit du travail, fiscalité...

Or l'Europe non seulement ne comporte pas d'échelon fédéral, mais elle fonctionne en régime de concurrence fiscale et sociale. Lorsque des pays ouvrent leurs économies les uns aux autres, il y a concurrence parfaite sur les marchés des biens, des capitaux et du travail, mais pas entre les Etats, à l'exemple de l'Irlande sur le plan fiscal ou de la France en matière de droit du travail. En la matière, il faut encore souligner que l'absence d'Etat fédéral, dont la principale fonction est une compensation partielle et minimale des déficits et des excédents d'épargne au sein d'une même zone monétaire, accentue la pression en faveur d'une convergence des économies ayant la même monnaie, que ce soit par l'obligation de mener des politiques budgétaires compatibles les unes avec les autres ou, en l'absence de dévaluations, d'ajuster les distorsions relatives de coûts du travail par leur réalignement « interne ».

La pratique européenne n'a pas confirmé la thèse d'inspiration keynésienne qui plaidait pour un système de coordination dans lequel les uns relancent (l'Allemagne) pendant que les autres réduisent (la France, l'Espagne...) leurs déficits. Les rares exemples de telles pratiques n'ont pas été concluants. La politique dite de la «locomotive », appliquée par le chancelier Schmidt à la fin des années 1970, a provoqué une hausse des prix et contraction du solde extérieur chez le pays qui relance (l'Allemagne) et n'a pas stimulé la croissance et la baisse du chômage chez ses partenaires. Dans les pays contraints de mener des politiques restrictives, les déséquilibres ne reposaient pas sur une insuffisance de demande mais sur un affaissement de la productivité et de la compétitivité. La France et l'Espagne en fournissent de très bons exemples : les déficits extérieurs que la crise a rendus brutalement insoutenables ne provenaient pas d'une insuffisance de demande chez le pays excédentaire (l'Allemagne) mais d'une détérioration marquée de leur compétitivité-prix entraînant, en l'absence d'ajustement du taux de change, un processus de désindustrialisation. Entre 2001 et 2008, le coût salarial unitaire du travail a reculé de $1 \%$ en moyenne annuelle en Allemagne du fait d'une progression des salaires de 1,8\%, inférieure à celle de la productivité $(2,9 \%)$. Il a progressé de $0,8 \%$ en France en raison d'une hausse annuelle moyenne des salaires de $3 \%$, supérieure à celle de la productivité (2,2\%). En Espagne, les coûts salariaux unitaires ont augmenté de $3 \%$ par an, en raison d'une hausse des salaires de $4,5 \%$ supérieure à celle de la productivité $(+1,5 \%$; Eurostat).

Ce double constat impose donc d'une part que les prix relatifs soient réalignés par des réformes de structure (réforme du marché du travail en Allemagne en 2003-2005, en Espagne en 2012, en Italie en 2015) et d'autre part, une véritable intégration des politiques budgétaires sur le modèle du semestre européen et du Traité de stabilité budgétaire (2012). Là encore, il convient de souligner que l'absence de niveau fédéral impose cette configuration alors que son existence n'impose pas une intégration aussi poussée, comme le montre l'exemple américain ou allemand.

Le troisième défaut de la construction d'origine porte sur l'absence d'union bancaire. Or ce sont les banques qui assurent le fonctionnement quotidien de la zone Euro. L'Europe est, à la différence des Etats-Unis, une économie d'inter- 
médiation bancaire : les banques fournissent les trois quarts des financements à l'économie. La question de l'Union bancaire fait ressortir trois dimensions du fonctionnement de la zone Euro que la crise a mises en évidence. D'abord la crise financière et des dettes souveraines est, à l'origine et avant tout, une crise de nature bancaire. Comme le souligne Nicolas Veron, "les banques sont au cœur de la crise européenne et l'ont été depuis le tout début. Contrairement à un préjugé tenace, la 'crise des subprimes' importée des Etats-Unis en 20072008 et la 'crise de la zone Euro' depuis 2010 forment une seule et unique séquence sans interruption parce que le secteur bancaire européen était resté en situation de fragilité systémique dès avant que les ennuis commencent en Grèce fin 2009 » (Veron, 2014).

Le point de départ des crises en chaîne de la fin des années 2000 n'est pas la chute de Lehman Brothers comme c'est souvent dit, mais la crise de liquidité des banques européennes à partir de l'été 2007 au moment où la banque IKB, fragilisée par un fonds subprimes à travers une structure irlandaise, a dû être secourue par les autorités allemandes, et où la BNP s'est trouvée dans l'impossibilité d'assurer la liquidité d'un de ses fonds. Comme l'indiquent Robert Raymond et Christian Bordes, " dès 2007, la paralysie du marché interbancaire, en particulier en Europe, due aux incertitudes sur la qualité du bilan des banques, a contraint les banques centrales à baisser les taux d'intervention et à approvisionner largement le système en liquidités " (Raymond/Bordes, 2014). A l'été 2007, pour enrayer la paralysie du marché interbancaire, la BCE est intervenue en passant par des appels d'offres à trois mois ( $L T R O$ - long term refinancing operation) pour conforter la trésorerie des banques. Fin décembre, elle leur a fourni 349 milliards $€$ au titre d'un appel d'offres exceptionnel (procédure de full allotment).

Un an avant la chute de Lehman Brothers, la BCE était donc confrontée aux premières manifestations de craquellement du marché interbancaire. La crise de la liquidité des banques européennes a ainsi déclenché un processus systémique de fragmentation du marché interbancaire européen qui mettait ouvertement en cause les canaux traditionnels (par les taux) de transmission de la politique monétaire. La crise de liquidité s'est muée en crise de solvabilité quand les banques du sud de la zone Euro ont vu, sous l'effet de la défiance des investisseurs à l'égard des dettes souveraines de leurs Etats, s'accumuler dans leur bilan des actifs dépréciés, augmentant du même coup la pression sur leurs Etats pour venir à leur secours. La malencontreuse déclaration à la suite du Sommet de Deauville du 18 octobre 2010 prévoyant une "participation adéquate du secteur privé " a fait ressortir la possibilité d'un risque de défaut sur les titres publics des Etats membres de la zone Euro alors que les investisseurs imaginaient devoir seulement supporter un risque de taux.

\section{La crise a permis à la BCE d'affirmer sa légitimité de banque centrale à part entière}

L'enchaînement des tourmentes monétaires, bancaires et financières de 2007 à 2012 a également fait ressortir l'obligation pour la BCE de clarifier et d'assumer les fonctions à part entière de banque centrale moderne, en liant l'objectif de stabilité des prix et celui de stabilité financière.

Dans les textes qui l'ont instituée, la BCE était vouée exclusivement à un objectif de stabilité des prix, interprété de plus de façon étroite. Cette conception repose sur l'idée que la stabilité des prix est obtenue par le contrôle de la masse monétaire. Dès le départ, la BCE s'est donné un objectif de croissance des prix de $2 \%$ à partir d'une croissance annuelle de la masse monétaire $\mathrm{M} 3$ de $4,5 \%$ fixé, lui, à partir de 2003. La crédibilité de cette politique est assurée par une banque centrale indépendante, sur le modèle de la Bundesbank, ce qui évite la tentation des gouvernements de manipuler la création monétaire à des fins politiques (voir Busch/Matthes, 2012).
Crise de liquidité des banques européennes dès avant la chute de Lehman Brothers

Puis crise de solvabilité

La fonction de stabilité financière découle de la mission de stabilité des prix ... 
lui re. mais lui reste subordonnée

Mutation de la BCE par nécessité

Crise de confiance

Réponse : baisse des taux, ...

... recours aux techniques quantitatives...
Si la BCE s'était tenue à l'écart de ses responsabilités en matière de stabilité financière, c'est bien l'Euro qui aurait volé en éclats avec une forte probabilité de diffusion du risque systémique à l'ensemble de l'économie européenne. En vertu du théorème d'Irving Fisher (1930) soulignant l'importance de sauver les banques en période de crise financière, il est impératif de fournir la liquidité nécessaire aux banques pour éviter les spirales déflationnistes : le manque de liquidité entraîne au nom de la course au cash un processus cumulatif de ventes d'actifs sur des valeurs toujours plus faibles. Dans une situation de paralysie des marchés, la fourniture de liquidités par la banque centrale évite qu'une crise de liquidité ne se transforme en crise de solvabilité.

C'est par nécessité que la BCE a opéré sa mutation. La crise et les secousses qu'elle a déclenchées dans les banques sont à l'origine de sa mue en banque centrale de plein exercice. Les mesures non conventionnelles adoptées au cours de cette phase visaient les banques en tant qu'institutions et ne comportaient pas de création monétaire puisqu'elles étaient stérilisées. La tension apparue en 2010-2011 avec la Bundesbank, marquée par la démission en février 2011 d'Axel Weber de la présidence de la banque centrale allemande, suivie quelques mois plus tard par celle de Jürgen Stark, le Chief Economist de la $\mathrm{BCE}$, ne reposait pas sur l'intention de rompre avec son credo monétaire mais bien sur la volonté d'assumer ses responsabilités. La mutation de la BCE a constitué probablement la condition nécessaire pour la pérennité et la stabilité de la monnaie unique. Fallait-il accepter une implosion du système bancaire européen qui aurait certainement mis fin à l'existence de l'Euro, au nom de la lettre de textes qui résultaient eux-mêmes des équivoques des gouvernements qui les avaient rédigés?

Avec la crise, la disparition de la confiance interbancaire et la paralysie des marchés interbancaires ont constitué la raison principale du déploiement des techniques dites «non conventionnelles ». La crise de confiance était telle fin 2007 que la BCE a dû négocier avec la Federal Reserve des accords de swaps pour obtenir les dollars que certaines banques européennes ne pouvaient plus obtenir de leurs correspondants. Dans des crises du type de celle que l'Europe a connu, l'assouplissement de la politique monétaire évite d'accentuer le caractère pro-cyclique des effets dépressifs de la crise. Il écarte l'incitation des banques à ajuster leur bilan par les crédits à l'économie, ce qui provoquerait une dégradation cumulative de la qualité de leurs actifs. Risque d'autant plus important que dans le même temps les politiques budgétaires doivent réduire les déficits publics et enrayer la montée de l'endettement des Etats.

Ce qui frappe, c'est le caractère massif des interventions à rebours du fine tuning des périodes de stabilité monétaire et financière. Baisse massive des taux d'abord. L'essentiel de la baisse des taux directeurs de la BCE s'est produit entre octobre 2008 et mars 2009 où ils ont été réduits de 4,25 à $1 \%$. Le mouvement s'est poursuivi. La dernière réduction de taux a eu lieu en septembre 2014 : le principal taux de la BCE ressort donc à 0,05\%.

Recours massif ensuite aux techniques quantitatives, ce qui constitue le fait le plus marquant de la transformation de la BCE. En mai 2009, la BCE est intervenue directement sur les marchés en mettant à la disposition des banques, pour éviter l'assèchement de leurs sources de financement, 60 milliards $€$ sous forme de covered bonds (obligations sécurisées). L'amplification des tensions sur les marchés et l'aggravation des risques de fragilisation des banques sous l'effet de l'emballement de la crise grecque ont conduit la BCE à intervenir sur les marchés obligataires privés et publics de la zone Euro. C'est le programme SMP (Securities Markets Programme) lancé en mai 2010. Les titres sont acquis sur le marché secondaire de la dette (il n'y a pas de financement des Etats mais refinancement des banques), et les opérations sont stérilisées : il n'y a pas de création monétaire. Fin 2012, l'encours de ces opérations atteignait 220 milliards $€$. 
La très forte dégradation de la situation en zone Euro fin 2011 a nécessité de la part de la BCE de franchir un nouvel échelon dans le déploiement de sa force de frappe. Fin décembre 2011, elle met en place un programme de LTRO (Long Term Refinancing Operation) à trois ans d'un montant de 489 milliards $€$. Une seconde opération a lieu en février 2012 pour un montant de 529 milliards $€$. L'élargissement considérable des moyens quantitatifs s'est accompagné d'un allongement significatif de la durée des opérations réalisées et d'un assouplissement des critères d'éligibilité des titres acceptés en garantie. L'éventail des actifs sous-jacents (Asset-Backed Securities - ABS) est élargi à des prêts hypothécaires, des prêts aux $\mathrm{PME}$, et les critères d'éligibilité sont ramenés à deux notations $\mathrm{A}$ au minimum à l'émission.

Enfin, dans le prolongement des déclarations de Mario Draghi affirmant le caractère irréversible de l'Euro et que tout serait fait - whatever it takes - pour l'assurer (26-07-2012), le successeur de Jean-Claude Trichet annonce le 6 septembre 2012 le programme OMT (Outright Monetary Transactions) ; la Bundesbank avait voté contre, et la Cour constitutionnelle de Karlsruhe été saisie par des groupes de contribuables. La Cour de Karlsruhe s'était elle-même retournée vers la Cour de justice de l'Union européenne (Müller-Graff, 2014) ; dans ses conclusions rendues le 14 janvier 2015, l'avocat général de la CJUE, rappelant que " cette situation exceptionnelle [i.e. la crise] a menacé la viabilité de la politique monétaire dont la Banque centrale européenne a la charge", estimait compatibles les opérations OMT avec les traités européens. Dans sa décision rendue le 16 juin 2015, la Cour européenne a confirmé la validité du programme OMT : "dans des conditions économiques telles que celles relevées par la BCE à la date du communiqué de presse, le Système européen de banques centrales (SEBC) pouvait valablement considérer qu'un programme tel que celui annoncé dans ce communiqué est apte à contribuer aux objectifs poursuivis par le SEBC et, partant, au maintien de la stabilité des prix ». Ce programme, qui remplace le programme SMP, prévoit, pour les Etats qui ont officiellement souscrit des programmes d'ajustement, l'acquisition sur le marché secondaire de leur dette publique de façon illimitée. Précisons encore que les opérations OMT avaient vocation à être stérilisées, comme la Cour européenne l'a relevé dans la décision précitée.

Ce mécanisme n'a jamais eu à être activé : les déclarations de Mario Draghi du 6 septembre 2012 affirmant le statut de prêteur en dernier ressort de la BCE sont d'une certaine manière venues parachever sa mue en banque centrale de plein exercice, ce qui a permis aux yeux des investisseurs de constater le caractère consubstantiel du lien entre l'Euro et la banque centrale qui l'émet.

Tant que la stabilité des prix a été assurée par l'intensification de la concurrence mondiale et que les marchés étaient capables de fournir une liquidité illimitée, la BCE a pu assurer ses missions sans avoir à mobiliser la plénitude de ses moyens et de ses fonctions : stabilité des prix et stabilité financière allaient de pair en pilotage automatique. Mais dès lors que les crises se sont produites, c'est bien à la banque centrale qu'incombait la responsabilité d'assurer l'accès des banques à la liquidité. C'est ce qu'a souligné, en pleine tempête, le gouverneur de la Banque de France, Christian Noyer, le 28 novembre 2011 à Tokyo, en rappelant l'obligation d'une banque centrale d'« assurer l'accès à la liquidité du système bancaire (ce qu'on appelle le rôle de prêteur en dernier ressort de la banque centrale) ». La fonction de prêteur en dernier ressort ne concerne ni les Etats ni les marchés financiers, mais les banques pour ce qui est du seul accès à la liquidité. Ce qu'a précisé Christian Noyer sur $L C l$ le 9 décembre 2011: " dans tous les pays, les banques centrales ne sont prêteuses en dernier ressort que des systèmes bancaires".

Dans la vision classique du rôle de la banque centrale, l'important est d'assurer l'objectif de stabilité monétaire qui passe par la stabilité des prix et par un accès aisé et suffisant des banques à la liquidité. En cas de défaillance des marchés, c'est bien à la banque centrale d'assurer la fonction de la liquidité. C'était d'ail-
... et annonce du programme OMT

La BCE, prêteur en dernier ressort 
Renversement d'objectifs

De la régulation de la liquidité bancaire... leurs son rôle traditionnel de refinancement direct des banques par l'escompte des effets de commerce auprès de ses guichets sous réserve de leur conformité à ses règles d'éligibilité avant l'essor des techniques d'open market.

La crise a donc constitué l'occasion pour la BCE d'affirmer par nécessité la légitimité de son rôle de prêteur en dernier ressort. Mais il en va autrement avec l'adoption d'une politique de Quantitative Easing depuis janvier 2015.

\section{La BCE et la « rupture » du Quantitative Easing : les risques de l'équivoque}

II existe une différence de nature entre le Quantitative Easing et les mesures non conventionnelles qui l'ont précédé. La première est une différence de doctrine d'emploi de la politique monétaire. On est passé d'une politique certes non conventionnelle, avant 2014-2015, considérant la stabilité des prix comme l'objectif de la politique monétaire, ce qui lui faisait obligation d'assurer la liquidité bancaire, à une convergence d'objectifs sur ceux de la Federal Reserve et de la Bank of England considérant que la stabilité des prix est subordonnée à un objectif de croissance et de chômage.

L'inflexion de doctrine était perceptible dès juin 2014. A l'occasion de l'annonce d'un nouvel abaissement des taux directeurs de 10 points de base réduits à $0,15 \%$, la BCE a annoncé la fin de la stérilisation des opérations SMP, ce qui manifestait sa volonté d'augmenter la masse monétaire de façon volontariste. Dans la perspective non de garantir un accès à la liquidité mais de créer une incitation à l'accroissement des crédits aux entreprises et aux ménages, la BCE a décidé d'effectuer des opérations de refinancement à plus long terme ciblées (TLTROs - Targeted Longer-Term Refinancing Operations) sur une durée de trois ans, jusqu'en septembre 2018. Ces opérations représentent un potentiel de 400 milliards $€$ de refinancements. Pour s'assurer que les banques emploieront ces ressources, les fonds non employés devront être remboursés.

Dans le même temps, Mario Draghi annonçait son intention d' "intensifier les travaux préparatoires relatifs aux achats fermes sur le marché des ABS [AssetBacked Securities] ... ayant pour actifs sous-jacents des créances vis-à-vis du secteur privé non financier de la zone Euro ». Le président de la BCE a exposé sa doctrine à Jackson Hole, le rendez-vous annuel traditionnel des banquiers centraux, quelques semaines plus tard, le 22 août 2014. II renoue avec l'arbitrage de Phillips selon lequel la politique économique devrait choisir entre le chômage et l'inflation, en soulignant que «le risque de 'faire trop peu' - à savoir que le chômage conjoncturel ne devienne structurel - l'emporte sur celui de 'faire trop' - soit des tensions excessives à la hausse sur les salaires et les prix ». En raison d'une reprise « uniformément faible» et d'« une demande morose », il estime que la « relance de la demande globale » doit accompagner les réformes de structure dont les effets récessifs sont ainsi amortis. La politique monétaire doit prendre leur relais et partiellement s'y substituer pour soutenir la demande. « Du côté de la demande, la politique monétaire peut et doit jouer un rôle central, ce qui se traduit actuellement par le maintien d'une orientation monétaire accommodante pendant une période prolongée. Je suis convaincu que l'ensemble des mesures que nous avons annoncé au mois de juin produira l'effet de relance escompté sur la demande ». Le raisonnement repose sur le fait qu'au-delà des facteurs exogènes comme le reflux du prix du pétrole, la baisse des taux d'inflation au-dessous de l'objectif de $2 \%$ à moyen terme provient d'une contraction de la demande induite par l'orientation restrictive des politiques budgétaires.

... à la régulation de la croissance

Mais Mario Draghi va plus loin. II prend ses distances avec la conception « rhénane » de la politique économique et plaide pour un policy mix, une combinaison des politiques budgétaire et monétaire, d'orientation keynésienne sur le modèle américain. La faiblesse de la demande impose une révision des objec- 
tifs assignés aux instruments de politique économique et particulièrement à la politique monétaire. Dans la conception classique énoncée par Tinbergen, c'est la politique budgétaire qui assure la régulation de la demande, et la politique monétaire qui pilote le taux d'inflation. Etant donné que les politiques budgétaires des Etats en déséquilibre consomment leurs marges de manœuvre pour leur désendettement et que ceux qui ont repris en main leurs finances publiques ne mobilisent pas leurs marges de manœuvre, la régulation de la demande globale ne peut provenir que d'une orientation accommodante de la politique monétaire. La baisse du taux d'inflation au-dessous de l'objectif de $2 \%$ fournit les marges de manœuvre nécessaires. Le président de la BCE observe en outre que les Etats qui ont rétabli leurs comptes publics (il s'agit de l'Allemagne) pourraient utiliser les limites permises par les traités pour le soutien de la demande en zone Euro. "II existe une marge de manœuvre pour mettre en place des politiques budgétaires plus propices à la croissance ». II faudrait, selon lui, une meilleure "coordination " des politiques budgétaires étant donné qu'il n'y a pas, en zone Euro, un seul budget mais dix-huit : "Une coordination plus forte entre les différentes orientations nationales en matière de politique budgétaire devrait en principe nous permettre de parvenir à une orientation budgétaire d'ensemble plus favorable à la croissance dans la zone Euro ».

Le renversement de paradigme monétaire a également porté sur le taux de change de l'Euro. Si la gestion du taux de change n'entre pas dans les attributions de la BCE, que ce soit par ses déclarations ou que ce soit la logique même du QE dans la volonté d'un accroissement massif de la création monétaire en Euros, Mario Draghi s'inquiétait des effets d'un taux de change trop élevé sur une inflation trop faible. "L'appréciation de l'Euro dans le contexte d'une inflation faible et de niveaux toujours bas d'activité économique (...) est un motif de préoccupation du point de vue du Conseil des gouverneurs ", a-t-il estimé en mai 2014. Et un mois plus tôt à Washington, il avait mis en évidence sa voIonté d'utiliser la création monétaire pour obtenir une dépréciation de l'Euro: "Si nous voulons que la politique monétaire reste aussi accommodante qu'elle l'est aujourd'hui, une poursuite de l'appréciation du taux de change [de l'Euro] pourrait nécessiter une action monétaire ». Dans cette perspective, la combinaison de la «forward guidance » et d'une création monétaire volontariste est destinée à rechercher à faire croître les prix intérieurs en important de l'inflation. Une conception étrange de la part d'un banquier central, car à la différence d'une hausse des prix provenant de celle des revenus, l'inflation par la baisse de l'Euro produit de l'appauvrissement des ménages qui paient plus cher les produits importés. Quoi qu'il en soit, le lien entre baisse du taux de change et création de monnaie a fonctionné puisque, dès l'annonce du programme de $Q E$ en janvier 2015, l'Euro a perdu environ $30 \%$ de sa valeur contre le dollar par rapport à mi-2014.

Le lien établi entre l'évolution baissière des prix, l'affaissement de la demande et la contraction des crédits au secteur privé a été conforté dans l'esprit de la $\mathrm{BCE}$ par la contraction de son bilan à partir de fin 2012. Les mesures non conventionnelles adoptées pour rétablir le fonctionnement normal des circuits de la liquidité bancaire se sont traduites par un fort gonflement de son bilan qui atteignait un plus haut fin 2012 à 3100 milliards $€$, soit $50 \%$ de plus qu'en 2009. A partir de 2013, avec le retour progressif à la normale sur les marchés, le rétablissement des comptes publics libérés de la pression des investisseurs et soutenu par une baisse des taux longs, ainsi que les premiers signes de reprise de la croissance, le bilan de la BCE s'est dégonflé pour revenir à environ 2000 milliards $€$. Ce qui est apparu comme une sorte de reflux progressif vers la normale et comme, au fond, une confirmation du succès de la gestion d'une période exceptionnelle, a été étrangement analysé comme une anomalie indésirable exerçant des effets dépressifs sur la demande. Lorsque les taux directeurs tendent vers zéro, c'est le bilan de la banque centrale qui prend le relais pour la gestion du taux d'intérêt réel. Dans la mesure où les banques ont en-

Action sur les taux de change 
Le passage au Quantitative Easing

Brouillage des objectifs de la politique monétaire trepris depuis début 2013 le remboursement des avances à trois ans dont elles ont bénéficié en 2010, la contraction du bilan de la BCE a joué l'effet d'un resserrement de la politique monétaire. Cette tendance a soutenu les taux d'intérêt réels en zone Euro et l'appréciation de l'Euro contre le Dollar. La BCE aurait pu estimer que conjuguée à l'amorce de la reprise économique, la réduction de taille de son bilan constituait une évolution normale et désirable. Mais le sentiment a prévalu qu'il fallait agir pour l'enrayer et le ramener à sa taille de fin 2012. A travers sa forward guidance initiée en 2013, destinée à diriger les marchés vers une perspective de taux quasiment nuls, la BCE a voulu réunir les conditions justifiant et permettant le passage au $Q E$.

Pendant tout le second semestre 2014, le président de la BCE a martelé le thème d'une inflation anormalement basse et d'une demande trop faible. En septembre, le principal taux directeur a été à nouveau réduit de 10 points de base à 0,05\% alors qu'en juin, Mario Draghi avait indiqué que les taux avaient atteint leur point bas. Dans le même temps, il a annoncé un programme d'achat d'ABS et d'obligations sécurisées (covered bonds) qui a débuté en octobre suivant, le montant visé étant de 500 milliards $€$. En novembre encore, au moment même où la reprise s'accélérait en Europe, il affirmait devant la presse que « depuis les mois d'été, les données obtenues et les résultats des enquêtes ont fait état dans l'ensemble d'un ralentissement de la dynamique de croissance dans la zone Euro ». Et d'insister sur la variation négative, certes en voie d'atténuation, des crédits aux entreprises dans les contreparties de la masse monétaire.

Annoncé par Mario Draghi le 22 janvier 2015, le programme de QE se caractérise par son ampleur et sa durée. Les techniques proprement dites de $Q E$ consistent à acheter des titres longs d'Etat pour obtenir une baisse des taux à moyen-long terme en raison de leur lien étroit avec l'investissement du secteur privé. Le QE est avant tout un achat massif de dettes publiques. Ses effets monétaires sont considérables puisque l'acquisition d'un titre public par la banque centrale revient à une annulation du titre émis à partir du moment où il entre dans son bilan. Cela revient à réduire le stock réel de dette publique au moyen d'une création monétaire massive.

Les programmes d'achat d'actifs publics s'ajoutent aux opérations effectuées sur des actifs privés. En cumulant les achats de titres adossés à des actifs $(A B S)$ et ceux d'obligations sécurisées (covered bonds) lancés auparavant, le total s'élèvera à 60 milliards $€$ par mois. Le programme devant s'étendre jusqu'en septembre 2016, c'est donc à peu près 1140 milliards € qui devraient être injectés dans l'économie sur l'ensemble de la durée du programme. Selon les indications disponibles, les achats d'obligations publiques étant réalisés en proportion du capital de la BCE, ils porteront sur 215 milliards $€$ d'obligations allemandes, 169 milliards $€$ d'obligations françaises, 147 milliards $€$ d'obligations italiennes, etc...

Les justifications d'un $Q E$ européen demeurent équivoques. D'abord l'Europe est bien différente des Etats-Unis où l'économie trouve ses financements majoritairement sur les marchés. En 2008, lors de son lancement, la banque centrale entendait prévenir une crise de liquidité. A un titre ou un autre, la zone Euro est loin d'offrir les conditions sur lesquelles s'est appuyée la Fed pour motiver son action. Les problèmes de liquidité des banques européennes ont été résolus par des techniques non conventionnelles non créatrices de monnaie et à un moment où il y avait péril sur les marchés interbancaires. Dans le même sens, les problèmes de rationnement de crédit (credit crunch) auxquels les entreprises, notamment les PME, étaient confrontées au moment où les banques concentraient leurs efforts sur l'assainissement de leurs bilans, avaient disparu pour l'essentiel au moment où le $Q E$ est intervenu.

Le risque de déflation ou d'une inflation évoluant en dessous de son objectif à moyen terme en raison de circuits bancaires défectueux ou de mauvaises conditions de transmission de la politique monétaire, repose sur des bases par- 
ticulièrement fragiles. A aucun moment, la zone Euro n'a été confrontée à un risque de déflation des prix dont on rappelle que, tenant à la difficulté de trouver de la liquidité, il incite à des ventes d'actifs entraînant des baisses cumulatives de leur valeur. Ce risque a été prévenu par les banques centrales dès l'origine de la crise, et la BCE, on l'a vu, a mobilisé son bilan de manière massive pour l'enrayer, en 2008 comme en 2011. Le niveau de l'inflation, au-dessous de sa tendance à moyen terme, résulte pour l'essentiel de la très forte baisse des prix de l'énergie. Compte tenu des pressions sur les coûts et les revenus par la concurrence internationale et dans les pays du Sud par le niveau encore élevé, bien que stabilisé, du taux de chômage, une politique massive de création monétaire aura des effets assez faibles sur la remontée des prix. Inversement, ses effets représentent des transferts de valeur substantiels en faveur des Etats et des marchés financiers. Au total, le $Q E$ aura probablement peu d'effets sur l'inflation - celle qui est mesurée par les indices des prix sur le marché des biens - mais beaucoup d'effets favorables aux Etats et aux marchés financiers.

Le $Q E$ peut s'interpréter comme une politique destinée non à résoudre les déséquilibres réels ou financiers de la zone Euro mais à sortir de la crise en recréant les conditions favorables à la formation de bulles. Bulles de dettes publiques en rouvrant aux Etats la voie à un endettement à coût réel nul ; bulles financières avec le report des injections massives de liquidités sur la valeur des actifs financiers, qu'il s'agisse des titres d'Etat ou de ceux émis par les sociétés. L'achat massif ferme de titres publics annoncé en janvier relève donc d'une logique tout autre que celles avancées jusqu'alors. Au-delà des considérations techniques de politique monétaire, les dirigeants de la BCE ont pris un risque lourd sur le terrain de la crédibilité et en définitive celui de la légitimité.

Le QE décidé par la BCE apparaît ainsi comme un « cadeau » aux hommes politiques et aux marchés financiers. Les premiers ne s'y sont pas trompés, qui ont salué avec enthousiasme la nouvelle orientation de la BCE puisqu'elle contribue à les décharger de leurs responsabilités dans la nécessité de mettre en place des réformes. Un politique monétaire aussi expansionniste avec ses taux d'intérêt aussi faibles permet certes d'alléger le coût des réformes, elle augmente aussi considérablement la désincitation à les engager. D'une certaine manière, elle subventionne les gouvernements qui renâclent à les entreprendre. Mario Draghi a certes souligné la nécessité de mener les réformes en raison du degré élevé de chômage structurel en zone Euro. Les réformes du travail notamment en Espagne (2012) et en Italie (2015) ont eu lieu. Mais à quoi bon les poursuivre ou, pour d'autres, les entamer, puisque la demande globale est prise en charge par la politique monétaire?

Pour les marchés financiers, la BCE est passée de sa fonction d'alimentation de la liquidité des banques sans création monétaire à celle de stimulation de bulles sur les marchés par la création massive de monnaie. Alors que la BCE non conventionnelle première manière avait consacré son énergie à permettre aux banques le nettoyage de leurs bilans, la BCE non conventionnelle seconde manière subventionne le retour à l'exubérance irrationnelle. L'économie mondiale a payé au prix fort à partir de 2008 l'éclatement des bulles spéculatives dont la formation a tenu pour beaucoup à la politique expansionniste de la Fed.

On sait aussi combien il est difficile pour une banque centrale de prendre le risque de «crever » une bulle. En cas de resserrement de sa politique monétaire, elle sera aisément accusée d'avoir provoqué une crise sur le marché obligataire. Comment gérera-t-elle donc la sortie du QE à partir de fin 2016 ? Chacun a pu noter les hésitations de la Fed sur la manière et le calendrier de sortie du QE. En Europe, depuis sa mise en œuvre opérationnelle en mars 2015, les rumeurs courent sur l'interruption du programme; la remontée des taux d'intérêt à long terme et du taux de change montrent bien l'incertitude sur son bien-fondé aux yeux mêmes des marchés. Qu'elle poursuive son programme ou qu'elle l'interrompe à un stade ou à un autre, la BCE assume donc d'ores et déjà un risque majeur de crédibilité comme Jens Weidmann, le président de la Bundesbank, l'a
Les effets désincitatifs du $Q E$ sur l'objectif de croissance

Ajournement des réformes par les Etats

Création de bulles sur les marchés

\section{Risque de \\ crédibilité}


Risque de légitimité

Avec la monétisation des dettes publiques, ...

... la BCE se substitue aux politiques budgétaires des Etats...

... au risque de perdre sa crédibilité souligné le 15 mai 2015 dans les colonnes du quotidien économique Handelsblatt: "Le fait de toujours remettre en question des décisions déjà prises porterait préjudice à la crédibilité du Conseil des gouverneurs de la BCE ».

A côté du risque de crédibilité de ses décisions, la BCE prend un risque considérable de légitimité en considérant que lui revient, certes dans un contexte de politiques budgétaires neutres ou restrictives, la prise en charge d'un objectif de régulation de la demande intérieure à des fins de stimulation de la croissance globale. Sur le terrain de l'opportunité, la justification avancée au nom de la faiblesse de la croissance et martelée tout au long de 2014, a été largement démentie par sa vigueur en zone Euro au $4^{\mathrm{e}}$ trimestre et en 2015.

La décision de la $B C E$, via le $Q E$, de monétiser partiellement la dette des Etats a en réalité modifié la nature de la politique monétaire qui entend ainsi se substituer à la politique budgétaire. On peut même estimer en la matière qu'elle tente d'assumer la fonction d'un échelon budgétaire fédéral dont la zone Euro est dépourvue. Jens Weidmann a raison de le souligner dans l'interview déjà citée au Handelsblatt : "Avec le programme de Quantitative Easing, les banques centrales de l'Eurosystème deviennent le principal créancier des Etats et la confusion entre politique monétaire et politique fiscale [i.e. budgétaire] continue de croître. Cela peut entraîner une augmentation de la pression politique sur les banques centrales lors des futures décisions de politique monétaire, d'autant plus que la volonté de procéder à des réformes est encore davantage affaiblie. » L'économiste français Patrick Artus met lui aussi en avant un risque majeur de perte d'indépendance: "Au total, l'indépendance et la crédibilité de la banque centrale sont menacées: si elle interfère avec les politiques budgétaires, les politiques structurelles, elle doit coordonner la politique monétaire avec d'autres politiques économiques, ce qui est incompatible avec l'indépendance » (Les Echos, 18-02-2015).

Sur le terrain des principes, la BCE, du fait du $Q E$, se retrouve en conflit d'objectifs et en situation de dépendance à l'égard des Etats. Car il s'agit bien d'une politique volontariste de création monétaire et d'une aide aux Etats par annulation partielle de leurs dettes. Le $Q E$ tourne radicalement le dos aux missions qui ont été confiées à la BCE qui n'a pas pour mission de faire du volontarisme monétaire ni de financer les Etats en les libérant de leurs dettes. Elle n'a pas à s'affranchir de l'objectif de stabilité des prix pour prendre en charge un objectif de croissance en se substituant à la politique budgétaire. Le $Q E$ redonne aux gouvernements les moyens de revenir aux facilités antérieures. La BCE se substitue aux politiques budgétaires : elle résout les excès de dettes publiques non pas en incitant aux réformes structurelles mais par leur effacement dans son bilan. Alors que la lutte contre l'inflation la poussait à affirmer son indépendance, la stimulation de la croissance en lieu et place de la politique budgétaire revient à consentir à la réduire.

La BCE s'est d'elle-même placée dans un dilemme dont elle aura toutes les peines à s'affranchir et qui se perçoit déjà dans les déclarations de Mario Draghi, comme cela est apparu lors d'une intervention à Washington le 14 mai 2015, où il a admis qu' « une période prolongée de politique monétaire accommodante peut, cependant, produire des effets collatéraux ». Le président de la $\mathrm{BCE}$ vise les effets sur l'allocation des ressources et la répartition des richesses. Le niveau très bas des taux, souvent proche de zéro voire négatif, favorise les emprunteurs au détriment des épargnants. Le risque existe qu'au lieu de désinciter à l'épargne et d'inciter à la consommation selon la conception néo-keynésienne habituelle, le niveau des taux proche de zéro induits par le $Q E$ ne génère au contraire une incitation accrue à l'épargne pour compenser la perte de revenus induite par la baisse de sa rémunération, particulièrement dans la perspective d'accumulation financière en vue des retraites.

Le passage d'une politique de neutralité monétaire calée sur les prix à une politique discrétionnaire de régulation de la croissance entraîne la BCE dans l'obli- 
gation d'arbitrer ses objectifs sur la répartition des revenus et des richesses au sein des sociétés européennes. En effet, une politique monétaire calée sur les prix est neutre sur les préférences en matière de redistribution de revenus ; alors que le pouvoir politique, sur la base d'un mandat démocratique, est libre d'afficher ses préférences. Mais en fonction de quels critères techniques et de quelle légitimité politique la BCE peut-elle alors justifier son action? Ainsi, elle s'est d'elle-même placée face à une alternative redoutable. Soit elle choisit de limiter les risques sur le terrain de l'efficacité en revenant partiellement sur son programme de $Q E$ pour amortir ses effets déstabilisants, et elle met à mal la crédibilité de ses décisions, ce qui paraît être un scenario peu probable. Soit elle poursuit l'expérience jusqu'à son terme en septembre 2016, et dans ce cas, elle prend le risque d'augmenter ses effets indésirables sur l'économie réelle tout en accroissant sa dépendance vis-à-vis des Etats et des marchés.

En définitive, le capital de crédibilité accumulé depuis sa création risque bel et bien d'être fortement entamé. La BCE ne peut pas vouloir, même en raison des déficiences de la gestion budgétaire en zone Euro, se substituer aux gouvernements. Au contraire, une politique durable de stabilité des prix exerce une pression permanente sur les déficits budgétaires et les réformes. Jens Weidmann n'a pas tort de relever, dans l'interview déjà citée, la moindre propension à réformer qui gagne la zone Euro. Et de rappeler que " la croissance durable dans la zone Euro ne peut pas être créée par un tour de magie de la politique monétaire. Il est indispensable que les gouvernements établissent les bases nécessaires ».

LES RISQUES D'UN EBRANLEMENT PROFOND DU PACTE FONDATEUR SONT GRANDS. La BCE est la pointe fédérale du projet politique européen ; elle est le cœur du pacte fondé sur une garantie réciproque convenue entre l'Allemagne et la France au lendemain de la chute du Mur de Berlin et de la disparition de l'URSS : pour la France, celle de l'ancrage à l'Ouest de l'Allemagne et de sa renonciation à la transformation en hégémonie politique de son leadership économique; pour l'Allemagne, la garantie qu'au nom même de son ancrage européen, le leadership économique allemand n'aurait pas à payer indéfiniment pour ses partenaires. Pas d'union de transferts pour l'Allemagne, pas d'obligation de contrôle de sa politique budgétaire par l'Allemagne ou/et l'Europe pour la France. Une union monétaire réduite aux acquêts ; une banque centrale dévolue à un rôle notarial. L'Euro avait donc initialement comme vocation première et fondamentale de neutraliser doublement le leadership économique allemand qui ne serait ainsi ni hégémonique ni servile.

La séquence de politique monétaire engagée depuis 2013-2015 ouvre une phase de relâchement des efforts nationaux d'assainissement et de réformes. Avec l'arrivée en novembre 2014 de Jean-Claude Juncker à la tête de la Commission et de Pierre Moscovici aux Affaires économiques, l'orientation générale de la politique économique et monétaire européenne a nettement pris ses distances avec les priorités antérieures de stabilité des prix et de réduction des déficits. Le relâchement manifeste de la pression européenne sur la politique budgétaire française illustre bien le nouveau cours économique et politique qui prévaut désormais à la Commission.

En termes politiques, la BCE de Jean-Claude Trichet, chantre de la désinflation compétitive, entendait s'affranchir des contradictions statutaires des débuts, fûtce au prix d'une "crise » avec la Bundesbank au nom de la pérennité de la monnaie unique. Mais alors que celle-ci était inséparable à ses yeux d'une convergence réelle de la zone Euro avec l'Allemagne, autant la BCE de Mario Draghi entend la soustraire à cet objectif.

Au bout du compte, l'Allemagne risque bien de se trouver dans la situation singulière de devoir assumer, par ses excédents d'épargne, la réassurance d'une union monétaire dont la contrepartie était à l'origine ses droits de contrôle de la politique monétaire de la banque centrale et des politiques budgétaires de ses partenaires. Situation d'autant plus étrange qu'en activant la «planche à bil- 
lets » européenne, la BCE est susceptible de mobiliser, sans qu'elle ait son mot à dire, l'épargne produite par l'économie allemande. La BCE alimenterait ainsi, même si c'est à son corps défendant, les conditions d'une crise de fond entre l'Allemagne et le reste de la zone Euro.

Bridée par ses principes de respect de l'indépendance de la BCE et sa ligne politique pro-Euro, l'Allemagne conserve une attitude mesurée en attendant de voir. La bonne tenue de la croissance en 2015 permet de reporter à plus tard les problèmes posés par les divergences de doctrine et de performances. Mais en désincitant aux réformes et à la réduction des déficits, le nouveau cours politique en vigueur à Bruxelles et à Francfort prend le risque de renforcer les disparités à l'intérieur de la zone Euro, notamment celles entre la France et l'Allemagne. La propension de la France à consommer s'est vue ainsi renforcée par le $Q E$ alors que la production, l'investissement et les exportations demeurent bloqués par son refus persistant d'engager les réformes de fond; en Allemagne, la baisse de l'Euro et des taux d'intérêt vont rendre encore plus compétitive une économie qui l'est déjà fortement. Une BCE focalisée sur les prix favorisait les forces renforçant la convergence de la zone Euro ; une BCE faisant le jeu de politiques budgétaires accommodantes risque au contraire d'entretenir les forces centrifuges. La BCE, qui craignait d'en avoir fait trop peu, pourrait bien être gagnée par le remords d'en avoir trop fait.

\section{Indications bibliographiques}

- ARTus P., « Et si Mario Draghi prenait trop de risques? », Les Echos, 18 février 2015

- Bindseil U., Clemens D., Zeuner J., Critique of accomodating central bank policies and the 'expropriation of the savers'. A review, BCE, Occasional Paper Series, mai 2015 (www.ecb.europa.eu)

- Busch B., MATHHES J., " Gouvernance de la zone Euro : comment prévenir la défaillance du politique ? - Le point de vue allemand », Regards sur l'économie allemande, n 105, été 2012

- DANIEL J.-M., La politique économique, Coll. Que sais-je ?, PUF, 2010

- «Die Bedeutung der makroprudentiellen Politik für die Geldpolitik », Deutsche Bundesbank Monatsbericht, mars 2015 (www.bundesbank.de)

- Draghi M., "Les récentes mesures de la BCE: efficacité et défis ", Cycle de conférences Camdessus, Washington DC, 14 mai 2015 (www.ecb.europa.eu)

- Dragh M., «Stabilité et prospérité dans une union monétaire », Project Syndicate, 02-01-2015 (www.ecb.europa.eu)

- Draghi M., Discours tenu le 26-07-2012 lors de la Global Investment Conference, Londres, 26 juillet 2012 (www.ecb.europa.eu/press/key/date/2012/html/sp120726.en.html)

- Hazouard S., Lasserre R., Uterwedde H., France-Allemagne : cultures monétaires et budgétaires, Editions du CIRAC, Cergy-Pontoise, 2015

- Kotz H.-H., " 60 ans de Loi fondamentale, 61 ans de Deutsche Mark », in BouRgeols I. (dir.), Allemagne. Les chemins de l'unité. Reconstruction d'une identité en douze tableaux, Editions du CIRAC, Cergy-Pontoise, 2011

- Le Heron E., "A quoi sert la Banque centrale européenne ? ", La Documentation française, Débats, RéflexEurope, Paris, 2013

- Matthius M., BLyth M. (dir.), The future of the Euro, Oxford University Press, Oxford, 2015

- MüLler-Graff P.-C., «BCE : monnaie, justice, démocratie - de Karlsruhe à Luxembourg », Regards sur l'économie allemande, $\mathrm{n}^{\circ} 112$, printemps 2014

- Perrut D., «L'opération vérité de la BCE sur les banques : une étape nécessaire mais non suffisante de la réforme de l'euro ", Fondation Robert Schuman, Questions d'Europe, n 332,10 novembre 2014

- Raymond R., Bordes C., "Les banques centrales, crises et défis ", Revue d'économie financière, $\mathrm{n}^{\circ} 113$, mars 2014

- Sinn H.-W., The Euro Trap. On Bursting Bubbles, Budgets, and Beliefs, Oxford University Press, Oxford, 2014

- "Ten years of monetary policy cooperation in the Eurosystem », Deutsche Bundesbank Monthly Report, avril 2008

- Veron N., "L'Union bancaire, un succès Européen », En Temps Réel, les Cahiers, septembre 2014

- Weber A., "Zehn Thesen zu zehn Jahren europäischer Geldpolitik », discours tenu à Bielefeld, le 23-06-2008 (www.bundesbank.de)

- Weidmann J., «Zur Rolle der Finanzstabilität für die Geldpolitik », discours tenu à Munich, le 2503-2015 (www.bundesbank.de)

- WeidmanN J., "Voraussetzungen für eine stabile Währungsunion », discours tenu à Bielefeld, le 13-10-2014 (www.bundesbank.de). 